Conclusion/Learning Outcomes Key learning from the initial development and implementation of the VRUs will be presented, considering the principles of the public health approach and UK government guiding principles for VRUs: collaboration; co-production; cooperative in data and intelligence sharing; counter-narrative development; and community consensus.

\section{E.005 FINDING SUBSTANCE USE DISORDER TREATMENT FACILITY OPENINGS: NATIONAL EXPANSION OF KENTUCKY'S LOCATOR}

Terry Bunn*, Tyler Jennings, Catherine Hines, Danita Coulter, Jodie Weber, Amber Kizewski. University of Kentucky, Lexington, USA

\subsection{6/injuryprev-2021-safety. 182}

Context FindHelpNowKY.org (FHNKY) is a drug overdose prevention website intervention developed and implemented in 2018 to timely link individuals to substance use disorder (SUD) treatment facilities with available openings in the Commonwealth of Kentucky. FHNKY is a near real time treatment locator where facilities update their openings on a regular basis. In February 2020, there were 607 facilities who participated on FHNKY and have updated their availability over 11,000 times; over 142,000 searches were performed. The website is used by health professionals, safety professionals, friends and family, as well as individuals themselves in search of SUD treatment.

Process The FHNKY website was rebranded as a national FindHelpNow (FHN) platform that includes standardized national search criteria, and specifically branded marketing materials.

Analysis A FHN peer-to-peer learning network was established for other states to utilize the national FHN platform and/or FHNKY strategies and approaches.

Outcomes Fourteen interested states attended an in-person meeting with multiple presentations on website development, and functionality in November 2019. In February 2020, five states and jurisdictions adopted the FHN platform and recruitment strategies, and memorandums of understanding have been established.

Learning Outcomes This initial development and implementation phase of a national standardized FHN platform will enhance the ability of any individual anywhere in the U.S., who seeks SUD treatment at their critical stage of readiness, to find a SUD treatment facility that accepts their form of payment (or fee-free), and provides necessary services such as the provision of medication for opioid use disorders and concurrent mental health treatment.

\section{A - Road - Programs, March 25, 2021}

\section{A.001 ONE-SIDEDNESS OF MOTORCYCLE HELMET USE: A CHALLENGE TO MOTORCYCLE SAFETY IN NEPAL}

${ }^{1}$ Puspa Raj Pant*, ${ }^{2}$ Lennart Hellmann, ${ }^{3}$ Hanhe Lin, ${ }^{2}$ Rüdiger Trimpop, ${ }^{4}$ Felix Siebert. ${ }^{1}$ University of the West of England Bristol, Bristol, UK; ${ }^{2}$ University of Jena, Jena, Germany; ${ }^{3}$ University of Konstanz, Konstanz, Germany; ${ }^{4}$ Technische Universität Berlin, Berlin, Germany

10.1136/injuryprev-2021-safety. 183
Background Mandatory motorcycle helmet use regulation is essential, but its enforcement is even more important for head injury prevention, especially in a country like Nepal with a high share of motorcycle traffic. We assessed the impact of one-sided motorcycle helmet use regulation in Nepal, where helmet use is mandatory, but only drivers are fined for not using a helmet, while passengers do not get punished.

Methods In a combined observational and questionnaire survey, knowledge about the helmet use regulation was assessed and adherence to the law evaluated. Data was collected at seven sites throughout the country, including rural and urban regions.

Results The observation of 2548 motorcycle riders reveals a dichotomy of motorcycle helmet use in Nepal. While $98 \%$ of drivers used a helmet, only less than $1 \%$ of observed passengers used a helmet. The questionnaire survey of 220 riders reveals a lack of knowledge about the existing mandatory helmet use for passengers, most probably due to inadequate enforcement of the helmet rule for them. The survey further reveals that helmets would be readily available for passengers, as over $50 \%$ have access to a helmet.

Conclusion In Nepal, the helmet use of motorcycle passengers is critically low. The sole existence of rules for mandatory helmet use is not enough, regulatory changes in the traffic-fine structure are necessary to increase helmet use and prevention of consequences of head injuries.

Learning Outcomes Educating motorcycle passengers about existing rules and benefits of using a standard helmet may support motorcycle safety in Nepal.

\section{A.002 COST OF MOTORCYCLE CRASH VICTIMS AT A PUBLIC TERTIARY HEALTHCARE FACILITY IN KARACHI, PAKISTAN}

${ }^{1}$ Asrar Ali* ${ }^{2}$ Adnan Hyder, ${ }^{1}$ Uzma Rahim Khan, ${ }^{3}$ Muhammad Ashar Malik. ${ }^{1}$ Aga Khan University Hospital, Emergency Medicine, Karachi, Pakistan; ${ }^{2}$ George Washington University Milken Institute School of Public Health, USA; ${ }^{3}$ Aga Khan University, Medical College, Community Health Sciences, Karachi, Pakistan

\subsection{6/injuryprev-2021-safety. 184}

Background Road traffic injuries account for the major proportion of injury and economic burden globally. However, motorcyclists are the most vulnerable group to sustain fatal and non-fatal injuries. The use of helmet is one of most acknowledged protective measure. However cost-saving ability of helmet use is inconsistent in the literature.

Aim To estimate the difference in the healthcare cost of motorcycle crash in those who were wearing helmet and those who were not wearing helmet in Karachi, Pakistan.

Methodology We conducted a cross-sectional study by using micro-costing method and out-of-pocket expenditure data collection for healthcare cost estimation in a public tertiary hospital of Karachi, Pakistan. We included 18 year and above victims of motorcycle crash both riders and pillions through consecutive sampling from the emergency department and grouped them in to helmeted and non-helmeted. Cost differences were compared using t-test.

Result We recruited 323 participants, 127 (39\%) were wearing helmet, while, 93 (60\%) were not wearing helmet at the time of crash. The helmeted group had $18 \%$ head injuries as compared to $46 \%$ in the non-helmeted. Helmeted group incurred a total healthcare cost of PKR.15855, whereas, nonhelmeted victims had a median healthcare cost of 
PKR.91229. Furthermore, the out-of-pocket (OOP) is lower in in the helmeted group (PKR.1250) compared to the counterpart (PKR.16800).

Conclusion We conclude that there are substantial cost implications of not wearing helmet and the traffic and road safety authorities should make sure that all motorcycle users including riders and pillions wear helmet not for their safety but also as a cost-saving approach to reduce burden on healthcare system.

\section{A.003 DRIVERS WHO CAUSE SERIOUS INJURY - CAN WE TARGET BEHAVIOUR CHANGE?}

James Nunn, Jo Barnes*, Emily Petherick, Andrew Morris. Loughborough University, Loughborough, UK

\subsection{6/injuryprev-2021-safety. 185}

Background Road safety campaigns tend to be nationally focussed, targeting all drivers, however these are general and often ineffective in reaching drivers causing serious road injury collisions. This study aimed to identify culpable drivers involved in collisions and consider the potential of delivering focused road safety campaigns to reduce serious injury on the roads.

Methods This study linked UK police data (STATS19) with hospital trauma data from the Trauma Audit Research Network (TARN) to identify serious (MAIS3+) injury collisions and the drivers involved for the county of Cambridgeshire (UK). These drivers were then assessed to be culpable, contributory or non-culpable for the serious injury collision. Additionally, geodemographic profiles were identified for the drivers using ACORN (A (UK) Classification of Residential Neighbourhoods) to establish if culpable drivers differed from non-culpable drivers. ACORN uses postcodes to differentiate areas by wealth (6 categories), available money (18 groups) and household description (62 types).

Results A total of 399 Cambridgeshire drivers were profiled, with 276 drivers considered culpable or contributing to the collision and 123 non-culpable. The ACORN categories and groups for the proportions of culpable and non-culpable drivers were similar. However, within the household types differences existed with culpable drivers having higher proportions of semi-skilled workers living in traditional neighbourhoods. In contrast non-culpable drivers had higher proportions of larger families living in rural areas.

Conclusion The findings suggest there are differences in specific household types. Using additional marketing tools, targeted road safety education campaigns could be aimed at culpable drivers living in these households

\section{A.004 MV CRASH CHARACTERISTICS AND MEDICAL CHARGES: FRONT-AND REAR-SEATED RESTRAINED AND UNRESTRAINED ADULTS}

${ }^{1}$ Joyce C Pressley*, ${ }^{2}$ Michael Bauer, ${ }^{2}$ Emilia Pawlowski, ${ }^{2}$ Sabana Bhatta, ${ }^{2}$ Leah Hines. ${ }^{1}$ Columbia University, New York, USA; ${ }^{2}$ Bureau of Occupational Health and Injury Prevention, New York State Department of Health, Albany, USA

\subsection{6/injuryprev-2021-safety. 186}

Background There are reports that historically higher mortality observed for front- compared to rear-seated adult motor vehicle (MV) occupants has narrowed. Vast improvements have been made in strengthening laws and restraint use in front-, but not rear-seated MV occupants suggesting there may be value in expanding the science on rear-seat safety.

Methods A linked 2016-2017 hospital and MV crash data set, the Crash Outcomes Data Evaluation System (CODES), was used to compare characteristics of front-seated $(n=130,761)$ and rear-seated $(n=6,641)$ adults aged 18 years and older involved in a MV crash in New York State (NYS). A primary enforced seatbelt law was in effect for front-seated, but not rear-seated occupants. Chi Square and linear regression use SAS 9.4.

Results Compared to front-seated occupants, those rear-seated were more likely to be unrestrained $(21.2 \%$ vs. $4.3 \%$, $\mathrm{P}<0.0001)$ and to have more moderate-to-severe injury/death $(11.9 \%$ vs. $11.3 \%, \mathrm{p}<0.0001)$. Compared to restrained rearseated occupants, unrestrained rear-seated occupants had higher moderate-to-severe injury/death $(21.5 \%$ vs. $7.5 \%$, $\mathrm{P}<0.0001)$ and 4 -fold higher hospitalization. Ninety percent (9 of 10) of rear-seated deaths were unrestrained. More than 95\% of ejections were unrestrained and had 7-fold higher medical charges. Hospital stays were longer, hospital charges higher and societal financial costs higher as the unrestrained were more frequently uninsured/self-insured/governmentinsured.

Conclusions These findings document increased medical charges and support the need to educate consumers and policymakers on the risks associated with adults riding unrestrained in the rear-seat.

Learning outcomes Describe crash outcomes and medical charges in front- and rear-seated restrained and unrestrained adult passengers

\section{A.005 REAR SEATBELT USE IN URBAN SOUTHEAST ASIA: RESULTS FROM MULTI-ROUND CROSS-SECTIONAL STUDIES}

${ }^{1}$ Edward Sutanto*, ${ }^{2}$ Nukbha Zia, ${ }^{1}$ Niloufer Taber, ${ }^{3}$ Fedri Rinawan, ${ }^{3}$ Indah Amelia, ${ }^{4}$ Piyapong Jiwattanakulpaisarn, ${ }^{2}$ Abdulgafoor M Bachani. 'Johns Hopkins Bloomberg School of Public Health, Baltimore, USA; ${ }^{2}$ Johns Hopkins International Injury Research Unit, Baltimore, USA; ${ }^{3}$ Department of Public Health, Faculty of Medicine, Universitas Padjadjaran, Bandung, Indonesia; ${ }^{4}$ ThaiRoads Foundation, Bangkok, Thailand

\subsection{6/injuryprev-2021-safety. 187}

Background Road traffic injury remains a leading cause of morbidity and mortality in Southeast Asia. Rear seatbelt use, a key behavioral risk factor, is understudied. We aim to estimate the prevalence and predictors of rear seatbelt use in Bandung, Indonesia and Bangkok, Thailand.

Methods Roadside observational studies were conducted across Bandung and Bangkok to provide a representative picture of behavioral risk factor prevalence in each city. Trained observers collected data from stationary vehicles at intersections during the daytime throughout the week. We computed descriptive statistics and conducted multivariable logistic analyses by city.

Results Eight rounds of observations were conducted between July 2015 to April 2019. 39,479 and 7,207 rear-seat passengers were observed in Bandung and Bangkok, respectively. Across all rounds, 4.2\% of rear seatbelt passengers used seatbelts in Bandung, compared to $8.4 \%$ in Bangkok. In both cities, males and adults, as compared to females and adolescents (aged 12-17 years), had higher odds of rear seatbelt use, as did passengers with a belted driver. Additionally, 